\title{
EVALUACIÓN FINANCIERA DE LOS PEQUEÑOS PRODUCTORES DE CAÑA DE AZÚCAR EN EL SUR DEL ECUADOR
}

\author{
Jossely Quishpe ${ }^{1}$, Liz Valle-Carrión ${ }^{2,3^{*}}$ Marco Heredia $^{4}$ \\ ${ }^{1}$ Titulación de Contabilidad y Auditoría, Universidad Técnica Particular de Loja, San Cayetano s/n, Loja-Ecuador. \\ ${ }^{2}$ Departamento de Ciencias Administrativas, Universidad Técnica Particular de Loja. \\ ${ }^{3}$ Instituto de Manejo Forestal, TUM School of Life Sciences Weihenstephan, Technische Universität München, Hans-Carl-von-Carlowitz-Platz 2 , \\ 85354 Freising, Alemania. \\ ${ }^{4}$ AgSystems, Ceigram, itdUPM, Centro de Innovación en Tecnología para el Desarrollo, Universidad Politécnica de Madrid (UPM), 28040, España \\ * Autor de correspondencia: lavalle1@utpl.edu.ec \\ Recibido: 2020/10/28 Aprobado: 2020/11/30 \\ DOI: https://doi.org/10.26621/XVI23.2020.12.A10.PUCESI.2550.6684
}

\section{RESUMEN}

El presente estudio versa sobre la producción de caña de azúcar de pequeños cañicultores rurales que trabajan en el cantón Catamayo de la provincia de Loja, en el Sur del Ecuador. El objetivo fue evaluar su desempeño financiero mediante costos reales e ingresos totales, para conocer la rentabilidad de la producción de la caña de azúcar que les genera a los pequeños productores bajo dos métodos de producción por cuenta propia y en coproducción.

Se evaluó la rentabilidad en bases a sus costos y gastos por los dos métodos de producción de los agricultores del sector y adicionalmente se aplicaron indicadores financieros, obteniendo los retornos generados por esta actividad agrícola. Se determinó en el modo de producción por cuenta propia un VAN de $\$ 7.762 .75$; asimismo, se obtuvo una TIR de $68 \%$, mientras que en el caso de coproducción generó un VAN de $\$ 4.693 .78$ y una TIR 55\%. Por lo tanto, en base a estos resultados, es notablemente superior en rentabilidad la alternativa de producir por cuenta propia para los agricultores de caña de azúcar.

Palabras clave: evaluación financiera, caña de azúcar, rentabilidad agrícola

\section{ABSTRACT}

This study is deals with production of sugarcane by small farmers who work in the Catamayo canton of the Loja province in southern Ecuador. The objective was to evaluate their financial performance through real costs, total income to know the profitability of the sugar cane production that It generates for small sugarcane producers under two modes of production on their own and in co-production.

The profitability was evaluated based on costs and expenses by the two farmers production methods of the sector. Additionally, financial indicators were applied obtaining the returns generated by this agricultural activity. NPV of $\$ 7$ 762.75 was determined in the own-account production mode, and an IRR of $68 \%$ was obtained, while in the case of co-production it generated an NPV of \$ 4693.78 , and an IRR of 55\%. Therefore, based on these results, the alternative of producing on their own for sugar cane farmers is notably higher in profitability.

Keywords: financial evaluation, sugar cane, agricultural profitability 


\section{INTRODUCCIÓN}

El cultivo de caña de azúcar mantiene relevancia mundial tanto para la alimentación como para la industria de bioenergía y productos derivados; se destaca por ser una valiosa materia prima industrial utilizada para producir una extensiva gama de productos, entre los cuales se destacan la azúcar orgánica y convencional, miel, aguardientes, etc. (Vera, 2006).

Brasil, China, India y Paquistán son los países con superficies mayores y más altos niveles de producción USAID (2011). Se estiman unos 25 millones de hectáreas sembradas en el mundo, principalmente para extracción de azúcar. Las diferentes industrias del mundo promueven más de 300 millones de empleos directos cada año (Castillo \& CINCAE, 2012).

América Latina y el Caribe se caracterizan por ser de las regiones principales que se dedican a la producción de azúcar, según OCDE-FAO (2019). Brasil produjo 32,2 millones de toneladas de azúcar en 2007 y entre $54 \%$ y $55 \%$ se transforman en etanol; en México, la producción de azúcar se pronostica en 5,7 millones de toneladas, lo que representa un aumento del 5,1\% respecto a la campaña 2006; en Argentina, la producción de azúcar debería ser superior por el incremento de la superficie sembrada. Asimismo, se prevé un incremento de la producción de azúcar en Ecuador, Colombia, Perú y Guatemala, mientras que está previsto una disminución en Cuba (Vera, 2006).

En Ecuador, la superficie cosechada de caña de azúcar ha mantenido una tendencia al alza, con una tasa de crecimiento a nivel nacional de 1,99\% entre 2015 y 2016 (ESPAC, 2016). De esta forma, la producción total nacional supera los 10 millones de sacos; actualmente el $98 \%$ es consumido en el mercado interno. Cabe señalar que alcohol y energía son generados a partir de los subproductos de caña de azúcar, cuyo cultivo se encuentra extendiéndose debido a la apertura de mercados, especialmente de biocombustibles, tal y como se indica en MAGAP (2019). Para abastecer la demanda en el país, existen seis grandes empresas moledoras de caña, como son: Valdez, San Carlos, Ecudos, Monterrey, lancem e Isabel María (Pérez, 2012). Potencialmente, la caña puede producir un promedio de $85.15 \mathrm{tm} / \mathrm{ha}$ (ESPAC, 2017).

De acuerdo a Díaz y Portocarrero (2002), el cultivo de caña de azúcar se desarrolla de forma favorable en suelos fértiles, profundos y sueltos; si se mantiene con riego, se logra conseguir acrecentar rendimientos mayores que en suelos sin riego. Puede producirse incluso en suelos marginales como los arcillosos y arenosos con un buen desagüe o drenaje; se logra aclimatar bien a los suelos con PH que va entre 4 a 8,3. El proceso de producción de caña de azúcar se sujeta a la implementación de recursos relacionados directamente con la producción agrícola, conocido también como ciclo de producción (Molina \& Olga, 2012). La producción de caña de azúcar cumple con etapas fenológicas en su crecimiento las cuales son: germinación, ahijamiento, rápido crecimiento, maduración y cosecha; esta última se lleva a cabo entre los 11 a 16 meses de la plantación; se quema la plantación para eliminar las malezas que impiden la cosecha (FIRA, 2010).

El precio al productor de caña de azúcar en pie, según los datos obtenidos del Ministerio de Agricultura y Ganadería, se encuentra en variaciones para el año 2016, desde USD 30.27 el mínimo y USD 34.87 el máximo con un promedio de USD 32.40, que está sobre el precio mínimo establecido; asimismo, para el año 2017, el precio mínimo fue de USD 30.28 y el máximo de USD 31.35, con un promedio de USD 31.43, por debajo al precio mínimo establecido (MAGAP, 2016).
Se debe señalar que, en el país, las zonas con mayor producción de caña de azúcar se encuentran localizadas principalmente en la región Costa. En el 2016, la provincia del Guayas alcanzó el 83.37\% de la superficie total cosechada de este producto. Por otro lado, en la Sierra, las provincias que tienen mayor importancia son Loja (con el 6.74\%) y Cañar (con $5.77 \%$ ) de la superficie cosechada (INEC, 2016).

Asimismo, en términos de producción, el 80,37\% del total de toneladas de caña de azúcar son producidas en Guayas, seguida por Loja con 8.98\% y Cañar con 5.51\% Respecto al rendimiento de toneladas por hectárea a nivel nacional, en la costa Guayas mantiene un rendimiento de 102.62 tm/ha y por otro lado, en la sierra, Loja tiene el mayor rendimiento a nivel nacional con el 162.24 tm/ha, Cañar con el 84.15 tm/ ha (ESPAC, 2016).

En este contexto, se pretendió evaluar la situación financiera para los pequeños productores en el cantón Catamayo, sector destacado en la producción de la provincia de Loja en cuanto al cultivo de caña de azúcar .

\section{MATERIALES Y MÉTODOS}

\section{Zona de estudio}

El cantón Catamayo se encuentra ubicado al sur de la República del Ecuador; pertenece a la zona 7. Los límites son, al norte, con la provincia del El Oro y el cantón Loja; al sur, con Gonzanamá; al este, con el cantón Loja; y al oeste, con el cantón Chaguarpamba, cantón Olmedo y cantón Paltas. Se halla ubicado a 1.232 m.s.n.m., con una extensión territorial de aproximadamente 651.27 km2; Catamayo se encuentra a 33,5 km de distancia de la provincia de Loja (Figura 1).
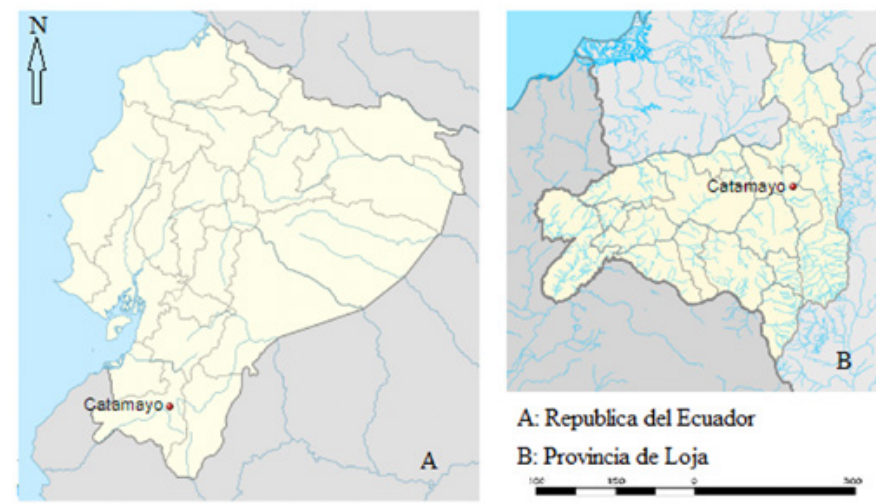

Figura 1. Ubicación de la zona de estudio: cantón Catamayo, Ecuador

\section{Levantamiento de la información}

Para la recolección de datos, se utilizó como instrumento de información una encuesta compuesta por dos secciones: 1) sección A, la cual consta de preguntas abiertas y cerradas para determinar las características agrícolas del sector y 2) sección $B$, que contiene preguntas cuyo propósito fue conocer el proceso de producción, costos que incurren en la producción desde la preparación de la tierra hasta cuando la caña está lista para ser vendida en pie, a fin de calcular cuál es la rentabilidad actual de la producción de caña de azúcar.

La selección de cañicultores se determinó a partir de los métodos de producción que se realizan en el sector de estudio 1) Cuenta propia, donde el agricultor con sus recursos financieros propios invierte en el cultivo de caña de azúcar; y 2) Coproducción, definido como producción realizada en común con un socio coproductor, quien cubre la parte de 
Ios costos de inversión. La encuesta fue aplicada a todos los agricultores del sector de estudio, de acuerdo a los registros del sector de estudio.

\section{Indicadores financieros}

En esta sección se detalla los indicadores financieros utilizados:

Tasa interna de retorno (TIR): es un indicador de rentabilidad, cuya relación es: a mayor TIR, mayor rentabilidad; esto se utiliza como uno de los criterios para decidir sobre la aceptación o rechazo de un proyecto de inversión, por lo cual la TIR se compara con una tasa mínima o tasa de corte, el costo de oportunidad de la inversión (si la inversión no tiene riesgo, el coste de oportunidad utilizado para comparar la TIR será la tasa de rentabilidad libre de riesgo); si la tasa de rendimiento del proyecto - expresada por la TIR- supera la tasa de corte, se acepta la inversión; en caso contrario, se rechaza (Cubillo, 2013).

Fórmula para el cálculo del TIR:

$$
T I R=\sum_{t=0}^{n} \frac{F n}{(1+i)^{n}}-I
$$

Donde:

$F_{n}=$ flujos de caja en cada periodo $n$

$\mathrm{I}_{0}=$ valor de desembolso inicial de inversión

I = tasa de costo de capital

$\mathrm{n}=$ número de periodos considerados

Valor actual neto (VAN): Es un procedimiento que permite calcular el valor presente de un determinado número de flujos de caja futuros, originados por una inversión. EI VAN depende solo de los flujos de efectivo proyectados provenientes del proyecto, así como del costo de oportunidad del capital y de los valores presentados que se pueden sumar porque se miden en dinero de hoy; es así que cualquier regla de inversión que no reconoce el valor del dinero en el tiempo no es razonable (Cubillo, 2013).

La metodología consiste en descontar al momento actual, es decir, actualizar mediante una tasa, todos los flujos de caja futuros del proyecto. A este valor se le resta la inversión inicial, de tal modo que el valor obtenido es el valor actual neto del proyecto.

La fórmula para el cálculo es la siguiente:

$$
V A N=\sum_{t=1}^{n} \frac{V_{F} t}{(1+T I R)^{t}}-I_{0}
$$

Donde:

$V_{\mathrm{F}} \mathrm{t}=$ Flujo de caja en cada periodo $\mathrm{t}$.

$\mathrm{I}_{0}=$ valor del desembolso inicial de la inversión.

$\mathrm{n}=$ número de periodos considerados

Punto de equilibrio: Se considera al punto en que los ingresos son iguales a los costos; es lo que se llama punto de equilibrio: en él no hay utilidad ni pérdida. Para calcular el punto de equilibrio, es necesario tener bien identificado el comportamiento de los costos, pues de otra manera es sumamente difícil determinar la ubicación de este punto. Para ello, se ha establecido que el punto de equilibrio se ubica donde los ingresos son iguales a los costos (Ramírez, 2013).
Se puede expresar algebraicamente de la siguiente forma:

$$
\boldsymbol{P E}=\frac{\mathrm{CF}}{\mathrm{P}-\mathrm{V}}
$$

Donde:

$\mathrm{IT}=$ Ingresos totales

$\mathrm{CT}=$ Costos totales

$\mathrm{P}=$ Precio por unidad

$X=$ Número de unidades a vender

$\mathrm{V}=$ Costo variable por unidad

$\mathrm{CF}=$ Costo fijo total en un tramo diferido

En el momento de calcular el punto de equilibrio, tanto en los costos variables como en los costos fijos, se deben incluir los de producción, administración, de ventas y financieros (Ramírez, 2008).

Relación Costo - Beneficio (B/C): es un indicador que mide el grado de desarrollo y bienestar que un proyecto alcanza; es conocido como índice neto de rentabilidad de un proyecto, que será rentable cuando la relación costo-beneficio sea mayor que la unidad (Lind, Marchal, y Wathen, 2012).

Es un cociente que se obtiene con la siguiente fórmula:

$$
\mathbf{B} / \mathbf{C}=\mathrm{VAI} / \mathrm{VAC}
$$

Donde:

$\mathrm{B} / \mathrm{C}=$ costo beneficio

$\mathrm{VAl}=$ Valor actual de los ingresos totales netos 0 beneficios $\mathrm{VAC}=$ Valor actual de los costos de inversión o costos totales

Rendimiento sobre capital (ROE): Es una medida de rentabilidad que tiene como característica dar a conocer el desempeño del renglón del resultado neto, con la finalidad de hacer conocer a los accionistas cómo les fue en el año o periodo económico; si el ROE es mayor que el ROA, la diferencia llega a ser el rendimiento (Ross, Westerfield, y Jaffe, 2012) La fórmula es:

$$
\mathbf{R O E}=\frac{\text { Utilidad neta }}{\text { Capital contable total }}
$$

Retorno de inversión (ROA): este indicador mide la eficiencia con la que una empresa genera utilidad a partir de los activos operativos promedios; así, mientras más alto es el porcentaje de rendimiento sobre activos operativos, ello se considera mejor (Bravo, Lambretón, y Márquez, 2007). El retorno de inversión es una medida de rentabilidad, cuya finalidad no es otra que dar a conocer cuál es la utilidad por dólar de activos. Esto se puede calcular de diferentes maneras, pero la más común es:

$$
\mathbf{R O A}=\frac{\text { Utilidad neta }}{\text { Total de activos }}
$$

Flujo de efectivo: Es aquel que informa sobre el origen y la utilización de los activos monetarios en las actividades de explotación; de este modo, los movimientos principales son los cobros y pagos provenientes por las actividades que constituyen la principal fuente de ingresos y 
gastos (Hervas, 2013). El flujo de efectivo proporciona información fundamental para poder evaluar la capacidad de generar dinero, así como la capacidad que tiene una empresa para pagar sus deudas, invertir, repartir dividendos y conocer cuándo se tiene la necesidad de obtener financiamiento. Asimismo, a través del flujo de efectivo, se puede determinar la calidad de la utilidad reportada (Espejo, 2007). Ross (2012) considera flujo de efectivo a la diferencia existente entre las unidades monetarias que entraron y las que salieron.

\section{RESULTADOS Y DISCUSIÓN}

En esta sección, se presenta la caracterización agrícola de los productores y la situación financiera a partir de los indicadores financieros ya referidos.

\section{Caracterización agrícola de los productores}

Los pequeños productores de caña de azúcar en pie del cantón Catamayo mantienen un promedio de superficie de terreno sembrado de 5,17 ha por cañicultor, porcentaje obtenido en base a los resultados obtenidos en las encuestas. Como se observa en la Figura 1, el $70 \%$ de los cañicultores tienen entre 1 a 5 ha, el $27 \%$ más de 5 ha, y solo el $3 \%$ de 0 a 1 ha. El $97 \%$ de los pequeños cañicultores cuentan con terrenos propios, el cual es destinado a la producción de caña de azúcar y solo el 3\% restante tienen terrenos en aparcería para la producción de caña de azúcar en pie. El 72\% de la producción de la zona mantiene rendimientos por hectárea de caña de azúcar en un rango de 120 a 150 toneladas y el $28 \%$ un rendimiento por hectárea sobre las 150 toneladas que va en relación con las condiciones climáticas, tierra y el cuidado de la producción. Asimismo, el Banco Central del Ecuador, en el año 2017, en el "Reporte de coyuntura sector agropecuario" da a conocer que la producción por hectárea en el cantón Catamayo para un pequeño productor se mantiene en $180 \mathrm{tm} / \mathrm{ha}$ (Tabla 1).

Tabla 1. Características agrícolas de los productores del cultivo de caña

\begin{tabular}{|c|c|c|c|}
\hline Variables & \multicolumn{3}{|c|}{ Producción de caña de azúcar } \\
\hline $\begin{array}{l}\text { Superficie de } \\
\text { producción (ha) }\end{array}$ & $\begin{array}{l}27 \%(0-10 \\
\text { ha) }\end{array}$ & ha) & 70\%(más a 5 ha) \\
\hline $\begin{array}{l}\text { Tenencia de } \\
\text { tierra }\end{array}$ & $\begin{array}{l}97 \% \text { terrenos } \\
\text { propios }\end{array}$ & & $\begin{array}{l}\text { o terrenos en apar- } \\
\text { cería }\end{array}$ \\
\hline $\begin{array}{l}\text { Rendimiento de } \\
\text { cultivo }\end{array}$ & $\begin{array}{c}72 \%(120-150 \\
\text { tn/ha) }\end{array}$ & & (mas de 150 tn/ha) \\
\hline
\end{tabular}

\section{Situación financiera del cultivo de caña de azúcar}

\section{Cálculo de rentabilidad}

Para realizar el análisis de rentabilidad de la producción de caña de azúcar / hectárea por los dos métodos de producción, se elaboró los flujos de efectivo para cinco cortes, considerando que es un cultivo permanente y no requiere ser sembrado después de cada cosecha.

En esta investigación, se tomaron en cuenta los ingresos y egresos generados hasta que la caña está lista para ser vendida en pie; según datos obtenidos de los cañicultores, la duración promedio de cada ciclo de producción de caña es de 15 meses en la zona de estudio. En base a lo antes mencionado, se procede a evaluar la rentabilidad agrícola por los dos métodos de producción.
Tabla 2: Flujo de efectivo de la producción de caña en pie, por cuenta propia por hectárea

Flujo de efectivo de caña en pie por cuenta propia USD

\begin{tabular}{lccccc}
\hline & Corte 1 & Corte 2 & Corte 3 & Corte 4 & Corte 5 \\
\hline Ingresos & $5.413,20$ & $5.307,11$ & $5.411,13$ & $5.517,18$ & $5.625,32$ \\
Egresos & $6.129,33$ & $1.356,67$ & $1.383,26$ & $1.410,38$ & $1.438,02$ \\
Utilidad & $\mathbf{- 7 1 6 , 1 3}$ & $\mathbf{3 . 9 5 0 , 4 3}$ & $\mathbf{4 . 0 2 7 , 8 6}$ & $\mathbf{4 . 1 0 6 , 8 1}$ & $\mathbf{4 . 1 8 7 , 3 0}$ \\
\hline
\end{tabular}

Fuente: Encuesta cañicultores 2018

En las tablas 1 y 2 se puede apreciar los flujos de efectivo de la venta de la producción de caña de azúcar en pie, por cuenta propia y por coproducción, los cuales tienen un crecimiento a partir del corte 2 en la utilidad, como consecuencia de que no generan costos de preparación de la tierra, semilla y siembra; por ende, disminuyen los egresos.

Para obtener la rentabilidad de la producción de caña de azúcar en pie, se aplicó los indicadores financieros VAN (Valor actual neto), TIR (Tasa interna de retorno), C/B (Costo beneficio) para cada uno de los métodos de producción. Cabe señalar que se ha considerado para los dos una tasa de descuento del 12\% (SENPLADES ,2014).

Tabla 3: Flujo de efectivo de producción de caña en pie, en coproducción por hectárea

\section{Flujo de efectivo de caña en pie por coproducción USD Corte 1 Corte 2 Corte 3 Corte 4 Corte 5 \\ $\begin{array}{llllll}\text { Ingresos } & 2.675,43 & 2.627,89 & 2.674,66 & 2.722,27 & 2.770,73\end{array}$

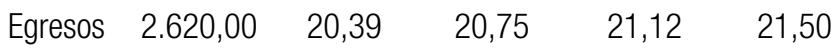 \\ $\begin{array}{lllll}\text { Utilidad } \quad 55,43 & 2.607,50 & 2.653,91 & 2.701,15 & 2.749,23\end{array}$}

Fuente: Encuesta cañicultores 2018.

En la tabla 3, se observa la rentabilidad generada para la producción de la caña de azúcar según cada uno de los métodos de producción. Para este cálculo, se tomó en cuenta el costo de hectárea de terreno natural (sin ninguna mejora), cuyo valor es de USD 907.00 de acuerdo a lo que reporta la Coordinación de avalúos y catastros del Gobierno Autónomo Descentralizado Municipal de Catamayo, y un estimado de USD 1.693.00 de costos en lo que concierne a la adecuación del terreno, costos de canales de riego, etc. de acuerdo a datos de expertos del sector obtenidos para esta investigación, lo que proporcionó resultados positivos en los dos métodos de producción.

Tabla 4: Desempeño financiero de la producción de caña en pie, en cuenta propia y coproducción por hectárea.

\begin{tabular}{lcc}
\hline & Cuenta propia & Coproducción \\
\hline VAN & USD $7.762,75$ & USD 4.693,78 \\
TIR & $69 \%$ & $55 \%$ \\
C/B & 1,66 & 1,94 \\
\hline
\end{tabular}

Fuente: Encuesta cañicultores 2018.

Se reportó un VAN superior en la producción de la caña de azúcar por cuenta propia como muestra la tabla 4, lo que significa que es más conveniente dedicarse a la producción de caña de azúcar en pie por cuenta propia, debido a que a mayor VAN mayor rentabilidad. 
En lo que respecta a la TIR, esta arrojó un resultado positivo para los dos métodos de producción de caña de azúcar en pie, ya que presenta resultados que están por encima de la tasa de descuento del $12 \%$ recomendada por SENPLADES (2014) para los proyectos de análisis de rentabilidad agrícolas; se considera por tanto que la producción por cualquiera de los dos métodos es aceptable. Sin embargo, la producción por cuenta propia tiene una rentabilidad mayor, con un 14\% por encima de la obtenida en coproducción.

Se estimó el cálculo del costo beneficio de una hectárea de producción de caña de azúcar en comparación con la producción de una hectárea de tomate (que es otro de los cultivos a los que se dedican los agricultores de la zona), para lo que se tomó en consideración 5 períodos y la inversión inicial. Los ingresos y egresos de la producción de tomate fueron tomados del reporte de coyuntura del Banco Central del Ecuador del 2017, resultados que fueron actualizados a valor presente, con la finalidad de conocer cuán conveniente es seguir produciendo caña de azúcar o cambiar de producción de acuerdo al análisis del costo beneficio.

De acuerdo al costo beneficio, se obtuvo un resultado de USD 1.38 en la producción de tomate, lo que significa que se obtuvo un beneficio de USD 0.38 por dólar invertido, USD 1.65 en la producción por cuenta propia de caña de azúcar con un beneficio de USD 0.65 y USD 1.94 en la producción por coproducción de caña con un beneficio de USD 0.94. A simple vista, se puede considerar que la producción de caña de azúcar en coproducción es más rentable. Sin embargo se debe tener en cuenta que, en coproducción, es el socio coproductor es quien asume la inversión, de ahí este resultado.

Por otro lado, una vez conocidos previamente el precio de venta, los costos variables y el porcentaje de participación de cada producto en las ventas, se ha podido encontrar el margen de contribución por cada método de producción.

Tabla 5: Contribución marginal por método de producción de caña de azúcar en pie.

\begin{tabular}{lcc}
\hline $\begin{array}{c}\text { Mezcla de contribución marginal por tonelada de caña } \\
\text { de azúcar USD }\end{array}$ \\
\hline & Cuenta propia & Coproducción \\
\hline Ventas & 29,95 & 29,95 \\
Costos variables & 11,40 & 13,05 \\
Contribución marginal & 18,55 & 16,90 \\
\hline Participación & $51.69 \%$ & $48,31 \%$ \\
\hline
\end{tabular}

Fuente: Encuesta cañicultores 2018.

Como se muestra en la tabla 5, para el cálculo de la contribución marginal se consideró un precio de venta de USD 29.95 por tonelada de caña de azúcar en pie, obteniendo como resultado una contribución marginal mayor en la producción de caña de azúcar en pie por cuenta propia con una diferencia de USD 1.65 en relación a la producción de caña de azúcar en pie por coproducción.

En la figura 2, se presenta el punto de equilibrio, el cual refleja la cantidad en toneladas por hectárea y en dólares que se necesita producir para cubrir los costos de producción por hectárea para no perder ni ganar; en el caso de la producción por cuenta propia, se necesita producir 69.32 tm/ha que equivale a USD 2.076.27 y para la producción en coproducción 54.89 tm/ha que equivale USD 1.644.00. Por tanto, los cañicultores deben producir una cantidad por encima del punto de equilibrio de tm/ha de caña de azúcar para obtener ganancias en la producción.

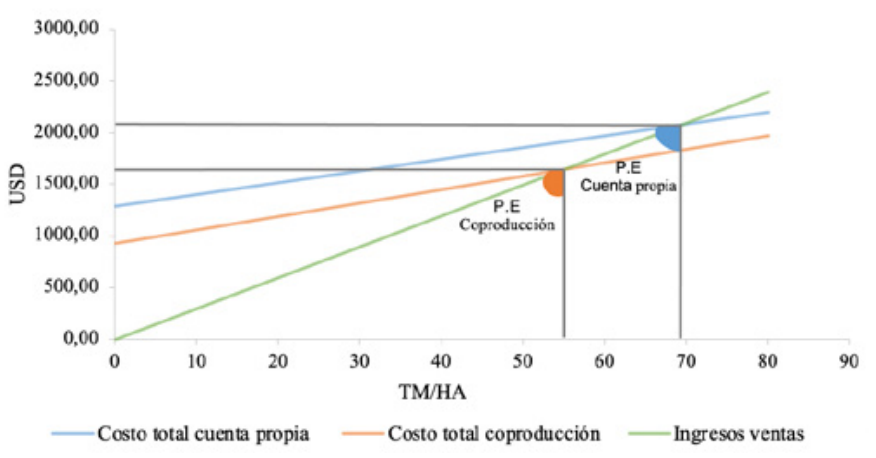

Figura 2. Punto de equilibrio de la producción de caña por coproducción y cuenta propia

Cabe recalcar que, para el análisis del punto de equilibrio, se tomó el $20 \%$ de los costos variables de la preparación de la tierra, semilla y siembra para el método de producción por cuenta propia, lo cual se sumó a los costos variables del corte 2.

Tabla 6: Cálculo del ROA Y ROE de la producción de caña de azúcar en pie por cuenta propia y coproducción.

\begin{tabular}{lll}
\hline & ROA & ROE \\
\hline Cuenta propia & $69 \%$ & $82 \%$ \\
Coproducción & $42 \%$ & $57 \%$ \\
\hline
\end{tabular}

Fuente: Encuesta cañicultores 2018.

Como se puede observar en la tabla 6 , para la producción por cuenta propia se obtuvo un ROA de $69 \%$, a diferencia de la producción por coproducción de 42\%; es decir, la producción por cuenta propia es más efectiva a la hora de utilizar sus activos para generar utilidades.

Los resultados obtenidos en el ROE para los dos métodos de producción de caña de azúcar son positivos, lo que significa que por cada dólar invertido en la producción por cuenta propia se obtiene un beneficio del $82 \%$ de rentabilidad y por cada dólar invertido por coproducción una rentabilidad del $57 \%$. Ello significa que el retorno sobre el capital es alto para los dos métodos de producción de caña de azúcar en pie.

Los resultados obtenidos en el ROE son superiores a los obtenidos en la aplicación del ROA. La comparación entre ROA y ROE permite conocer que los dos métodos de producción de caña de azúcar pueden financiarse por fuentes externas para el crecimiento de la producción, ya que la tasa de interés -de acuerdo al BanEcuador para este tipo de financiamiento- es de $11,57 \%$, cifra por debajo de los rendimientos del ROA.

\section{Discusión}

Del $100 \%$ de la producción de caña de azúcar, el 73\% de la producción se destina a la producción por cuenta propia y el $27 \%$ en contratos por coproducción con un ingenio local; los productores se abastecen de recursos financieros el $76 \%$ con capital propio, el 18\% crédito bancario y $6 \%$ crédito familiar. Este comportamiento es debido a que el acceso a financiamiento externo resulta difícil para los pequeños cañicultores, ya que los retornos de la inversión son tardíos, los que no permite soportar los paquetes crediticios de las instituciones bancarias; como consecuencia, es difícil incrementar la inversión para mejorar su producción. Se destaca que los cañicultores que trabajan por contratos de coproducción presentan un resultado más atractivo para la producción de caña de azúcar. 
La producción de una hectárea de caña de azúcar en el cantón Catamayo tiene ingresos de USD 5.400, de los cuales el 57\% se destina para cubrir los costos incurridos en la producción de caña. Así también, en el estudio de la cadena de valor de los cañicultores del cantón Catamayo en el 2017, el costo aproximado de la siembra de una hectárea de caña de azúcar es de USD 1.168,86 sin tomar en cuenta un costo muy importante como es la siembra (BCE,2017). De acuerdo con este estudio, los costos promedios de producción de caña de azúcar por hectárea para el proceso de siembra y cuidado de la caña en cuenta propia es de USD 3.559,26 y en coproducción de USD 4.028,37; por tanto, resultan más elevados los costos incurridos en la producción en contrato de coproducción, con un promedio de 12,46\% en relación con los costos de producción por cuenta propia.

Los costos más significativos que incurren en la siembra de caña de azúcar del primer año son la preparación del terreno (en un 37,29\%), monto que puede variar de acuerdo a las condiciones del terreno; los costos por semilla fueron $10,15 \%$, siembra $14,95 \%$ y riego $14,29 \%$, lo cual puede variar de acuerdo a la edad de corte de la caña. Sin embargo, los cañicultores que están en coproducción no realizan ninguna inversión para mantener el cultivo, debido a que los costos los cubre el socio coproductor.

El rendimiento promedio de caña de azúcar es de 165 tm/ha, sin dejar de mencionar que los cañicultores de la zona de estudio cuentan con el mayor rendimiento por hectárea a nivel nacional (ESPAC, 2016).

La utilidad promedio para la producción por cuenta propia es de USD $3.950,43$ y la utilidad promedio en contratos de coproducción USD $2.607,50$, manteniendo una diferencia promedio del $33,99 \%$. No obstante, los cañicultores dedicados a la producción de caña deben esperar entre 15 a 16 meses para obtener los primeros retornos; por esta razón, el $52 \%$ de los cañicultores tienen la necesidad de dedicarse a otras actividades que mitiguen sus gastos para subsistencia.

En relación a este estudio, el método de producción más rentable es la producción por cuenta propia de acuerdo a los indicadores aplicados, los cuales arrojaron una TIR en la producción por cuenta propia de 69\% y por coproducción de 55\%, resultados que están por encima de la tasa de descuento del $12 \%$, tasa que recomienda SENPLADES; el VAN por cuenta propia se establece en USD 7.762,75, a diferencia del VAN obtenido por coproducción (de USD 4.693,78), razón por lo cual resulta conveniente realizar el cultivo por cuenta propia en virtud de estos resultados.

Por otra parte, los cañicultores de la zona deben producir cantidades superiores al punto de equilibrio, hecho que va permitir solventar los costos por hectárea de producción, pues en cuenta propia se debe producir mínimo 69,32 tm/ha y en la producción por coproducción mínimo 54,89 tm/ha, con lo cual les será posible solventar los costos de producción.

Es importante tener presente que el costo de riego de la caña de azúcar es uno de los costos más significativos de la producción en relación al segundo periodo de corte de la producción en cuenta propia con un promedio de $30,79 \%$ y coproducción $47,30 \%$, el cual va relacionado directamente con la edad en que es cortada la caña; por tanto, una manera de disminuir el costo (sin que no se vea afectado el rendimiento y calidad de la producción) es que las cosechas no esperen tanto tiempo adicional una vez que la caña haya cumplido su ciclo y pueda o esté en condiciones de ser cosechada.

Es relevante y pertinente que el Estado busque mecanismos con los que ofrecer paquetes crediticios para producción en pequeña escala y con esto lograr motivar a los pequeños productores que abastecen el mercado interno y no tener la necesidad de buscar otras alternativas de financiamiento .Es necesario también que se genere políticas para proteger y mitigar los impactos de externalidades como sobreproducción mundial o fluctuaciones en el valor de la tonelada de caña de los ingenios, que afectaría gravemente los costos de producción y rendimientos de las cosechas.

\section{CONCLUSIONES}

Se concluye que existe rentabilidad en la producción de caña de azúcar y que la alternativa más atractiva o rentable para los cañicultores es por cuenta propia ya que presenta un resultado mayor en cuanto al VAN con el 39,54\%; en relación a la producción en coproducción y en los indicadores de rentabilidad presenta mayores resultados con una diferencia de, TIR superior con 19,11\%, ROA superior de 39,13\% y ROE 32,92\% superior. Se exceptúa el margen operacional y el costo beneficio, ya que estos indicadores se los obtiene a partir de la diferencia de ingresos menos egresos y en el caso de la producción por coproducción es el socio coproductor quien asume los costos de producción.

\section{REFERENCIAS BIBLIOGRÁFICAS}

Álvarez , A., y Sánchez, B. (2014). Formulación y evaluación de proyectos agrarios. En Agrarias. Colombia: Ecoe.

BCE. (2017). Reporte de conyuctura sector agropecuario. https://contenido.bce.fin.ec/documentos/PublicacionesNotas/Catalogo/ Encuestas/Coyuntura/Integradas/etc201604.pdf.

Bravo, M., Lambretón, V., y Márquez, H. (2007). Introduccion a las finanzas. México: Pearson Educación

Castillo, R., y CINCAE. (2012). Caña de azúcar: Cultivo para la sostenibilidad. https://elproductor.com/editorial-del-mes/cana-de-azúcar-cultivo-para-la-sostenibilidad/.

Cubillo, M. (2013). Valor Actual Neto. Obtenido de http://miguelbarrachinacubillo.jimdo.com/valor-actual-neto-van/

ESPAC. (2016). Encuesta de superficie y producción agropecuaria continua. http://www.ecuadorencifras.gob.ec/documentos/ web-inec/Estadisticas_agropecuarias/espac/espac-2016/Informe\%20ejecutivo\%20ESPAC_2016.pdf.

ESPAC. (2017). Encuesta de superficie y producción agropecuaria continua. http://www.ecuadorencifras.gob.ec/documentos/ web-inec/Estadisticas_agropecuarias/espac/espac-2017/Informe\%20ejecutivo\%20ESPAC_2017.pdf.

FAOSTAT. (2018). Cultivo. http://www.fao.org/faostat/es/\#data/QC. Recuperado el Abril de 2018

OCDE-FAO PerspectivasAgrícolas 2019-202. Annual.ISSN: 22184376 (online)

GAD. (2014). Gobierno autónomo descentralizado Catamayo. http://www. gadmunicipaldecatamayo.gob.ec/datos-generales-del-gad-3/.

GAD. (2015). http://www.gadmunicipaldecatamayo.gob.ec/catamayo/. GAD MUNICIPAL CATAMAYO.

INEC. (2010). Instituto nacional de estadísticas y censos . http:// www.ecuadorencifras.gob.ec//encuesta-nacional-de-ingresos-y-gastos-de-los-hogares-urbanos-y-rurales/.

INEN (2017). Instituto Ecuatoriano de Normalización. http://www.ecuadorencifras.gob.ec//encuesta-nacional-de-ingresos-y-gastos-de-los-hogares-urbanos-y-rurales/

Lind, Marchal, y Wathen. (2012). Estadística Aplicada a los Negocios y a la Economía. México: Mc Graw Hill.

MAGAP. (2016). La política agropecuaria ecuatoriana: hacia el desarrollo territorial rural sostenible: 2015-2025I Parte ISBN: 978-994222-019-6@ Ministerio de Agricultura, Ganadería, Acuacultura y Pesca 2016 
MAGAP. (2019) (MAGAP). Programa Nacional de Agroenergía. Ministerio de Agricultura, Ganadería, Acuacultura y Pesca

Mete, M. R. (2014). Valor actual neto y tasa de retorno: su utilidad como herramientas para el análisis y evaluación de proyectos de inversión. Fides et Ratio-Revista de Difusión cultural y científica de la Universidad La Salle en Bolivia, 7(7), 67-85.

Molina, \& Olga. (2012). El sistema de acumulación de costos. Su importancia y pertinencia en el sector agrícola merideño. $A c$ tualidad Contable Faces, 15(24). http://www.redalyc.org/ pdf/257/25722697004.pdf.

Ross, Westerfield, y Jaffe. (2012). Fundamentos de finanzas corporativas. México: Mc Graw Hill

Ramírez, D. (2008). Contabilidad administrativa. México: Mc Graw-Hill.

Pérez. (2012). Variedades y tecnologías en caña de azúcar. http://www. revistaelagro.com/wp-content/uploads/2012/08/ElAgro206.pdf.

Sánchez. (2002). http://www.redalyc.org/html/280/28011673008/. En I. d. rentabilidad, herramientas para la toma decisiones financieras.

Vera, I. A. (2006). Cultivo caña de azúcar.

https://bibliotecadeamag.wikispaces.com/file/view/Cultivo+de+Ca\%C3\%B1a+de+Az\%C3\%BA car-1.pdf. 